\title{
COMMENT: PHONON SPECTRUM OF MOLECULAR CRYSTALS FROM EXCITON SIDEBANDS ${ }^{\ddagger}$
}

\author{
R. KOPELMAN, F.W. OCHS and P.N. PRASAD \\ Department of Chemistry, The University of Michigan, \\ Ann Arbor, Michigan 48104, USA
}

Received 18 March 1974

Revised manuscript received 28 J une 1974

\begin{abstract}
Phonon sidebands of excitons in molecular crystals with negligibly small excitation exchange interactions, including isotopic guest systems, are net, in general, expected to be predominantly weighted by translational modes that are predictable from Brillouin zone centre $(q=0)$ ones. Some phonon sideband data on naphthalene, hexamethylbenzene, etc. are discussed and argued to map the extent of the phonon density-of-states as well as some of the $q \neq 0$ singularities.
\end{abstract}

In a previous paper [1] we have suggested that the phonon sidebands of exciton transitions with negligibly small excitation exchange interactions, specifically for the case of guest emission in isotopic mixed molecular crystals, are described by:

$I_{1}^{f}(\omega)=I_{0}^{f} G_{\mathrm{op}}^{f}(\omega)$

where $I_{1}^{f}(\omega)$ is the one-phonon contributed intensity, $I_{0}^{f}$ the zero-phonon intensity (which is assumed to be much larger than the integrated one-phonon intensity in order to justify the neglect of multiphonon contributions) and

$G_{o \mathrm{p}}^{f}=(12 N)^{-1} \sum_{S, q} C_{S, q}^{f} \delta\left(\omega-\omega_{S, q}\right)$

where $C_{S, q}^{f}$ is an exciton-phonon coupling amplitude, dependent on the excitation $f$ (which causes a change in force field) as well as on the phonon reduced wave vector $q$ and the branch polarization in $\operatorname{dex} S$. It was further argued that the couplins amplitude $C_{S, q}$ is not expected to have singularities as a function of energy and that the singularities of the amplitude weighted density-of-states $G_{\text {op }}^{f}$ should re. present the singularities of the density-of-states, $g(\omega)$,

Supported by NSF Grant GH-32578X. where

$g(\omega)=(12 N)^{-1} \sum_{S, q} \delta\left(\omega-\omega_{S, q}\right)$

It was further argued experimentally that for a number of vibronic sidebands of isotopic mixed naphthalene crystals the ten or more observed peaks map some of the singularities in the phonon density-ofstates function, without necessarily being limited to $q=0$ points, in reasonable agreement with theoretical calculations [2], considering their approximate na. ture, and with neutron inelastic incoherent scattering data [3], considering their limitations due to multiphonon scattering effects; in addition to hydrogen motion weighting as well as temperature and resolu. tion effects.

Recently, our above work was criticized by Reynolds [4]. He concludes that (I) the phonon sidebands on excitons in molecular crystals do not measure at all closely the true density-of-states but (II) a function related to some of the Brillouin zone center translational mode frequencies. We do essentially agree with the first part (I), as we only claimed to map son!e singularities in the density-of-states, and pointed aut that the true density -of-states can only be derived from there by a "Bootstrapping" technique, 
involving improved model calculations (which in turn also rely on other measurements like Raman, infrared, neutson scattering, etc.). However, we disagree strongly with the second part (II) of Reynolds' claim [4], i.e., that the above sidebands measure a function related to some translational frequencies which may "often" be predicted from data on the Brillouin zone center ( $q=0)$ translational modes.

Theoretically, in order to justify his claim (II), Reynolds argues that the change in the pairwise van der Waals force upon excitation of one molecule is proportional to the change in the square of the intermolecular electronic overlap between the members of the pair (he admits that to be crude). Furthermore, he assumes the transition electron density to be essentially spherically symmetric (for $\pi-\pi^{*}$ and apparently also $n-\pi^{*}$ transitions). In addition, he assumes the molecular elcction density itself to be spherical (this for the molecules: naphthalene, paradichlorobenzene and pyrazine). We find the above arguments difficult to accept. In addition, they lead to inconsistencies: (a) A spherically symmetric molecular electron density, in addition to a spherically symmetric transition. electron density, seems to imply not enly a zero electron-rotational phonon coupling, but also a zero rotational phonon frequency! This is in strong contradiction to the observed $\boldsymbol{q}=0$ rotational phonons in the Raman spectra of naphthalene [5], paradichlorobenzene [6], etc. which spread up to and above $100 \mathrm{~cm}^{-1}$. (b) A spherically symmetric molecule (and even the real naphthalene, pyrazine or paradichlorobenzene molecule, even in its crystalline site) preserves a center of symmetry (with respect to inversion) and, therefore, could not have a nonvanishing coupling between a $\mathrm{g}$-u electron transition (as in Reynolds' quoted examples) and an unyerude orie-phonon translational mode $(q=0)$. Furthermore, by implicitly assuming [4] a separation of rotation from translation for all $q$; and by explicitly claiming a strong branch dependence of intensities, this ungerade symmetry should apply for all $q$. This would result in a zero intensity for all the one-phonon translational sidebands, and especially for $q \approx 0$. This contradicts again the claim that the latter would account for the most intense sideband peaks.

On the experimental side, our quoted naphthalene data [i] have to be pushed very hard, so as to uniquely agree with the published far-infrared frequencies
[7] \# as claimed by Reynolds [4]. These data [1] do agee as well, if not much better, with the Raman frequencies, implying the importance of coupling with rotational modes (which still we never clairned), and also agree about as well with the neutron scattering data and the theoretical density-of-states peaks [1]. Faced with $10 \mathrm{~cm}^{-1}$ uncertainties in a crowded region we did not attempt [1] an assignment. We doubt as well Reynolds' assignment [4]. Most importantly, we refuse to accept an interpretation which implies that the naphthalene structure with more than ten distinct peaks derives the bulk of its intensity from the only thee possible nonzero $q=0$ translational frequencies (we still believe that some of these peaks may be related to essentially translational modes). Furthermore, we do have additional data [9] to show that our reported isotopic (dilute) mixed crystal naphthalene phonon sidebands are very mildly polarized. This makes less certain Reynolds expectation of "an extremely stiong depeidilence of $C_{S, q}^{f}$ on branch $S$ " (see eqs. (8) and (14) of ref. [II). However, we do not exclude, in general, the possibility of the existence of such sidebands in molecular crystals (but they would not necessarily be our favorite choices for a phonon singularity mapping investigation).

For pyrazine, both "phonon sidebands" quoted by Reynolds [4] (57 and $82 \mathrm{~cm}^{-1}$ have been demonstrated to be artifacts, related to triplet defect origins (with different life times and very different temperature characteristics), by Azumi and Nakano [10]. The remaining broad, single humped distribution certainly fits much better the density-of-states and the incoherent neutron scattering, as actually quoted by Reynolds [4] rather than the sharp peaks he expects to match the IR peaks. Moreover, the Raman (by Ito [11]) frequencies quoted there [10] are (at $4 \mathrm{~K}$ ) 56 and $86 \mathrm{~cm}^{-1}$, that is, too close for comfort to the quoted [4] infrared frequencies ( $77 \mathrm{~K}$ ) of 55 and 80 $\mathrm{cm}^{-1}$. For paradichlorotenzene, it is not obvious whether the weak and diffuse phonon sidebands and the far-infrared data which Reynoids compares [4] are taken for the same crystal phase $[3,12]$. In addition; the $90 \mathrm{~K}$ Raman data [13] (monoclinic; most likely $\alpha$ phase: $32,57,64 \mathrm{~cm}^{-1}$ ) seem to fit about as well as the far-infrared data...

\# Moreover, they imply that the $54 \mathrm{~cm}^{-1}$ peak is ar artifact. It was indeed shown as sich by Bree and $K y d d[8]$. 
Finally, we have investigated with some care the phonon sidebands of hexamethylbenzene [14]. This molecule is certainly not less spherical than naphthalene, paradichlorobenzene or pyrazine. As the in. vestigated crystal phase has only one molecule per primutive unit cell one would expect, following literal. ly Reynolds' approach [4], to have exciton-phonon sidebands exhibiting only acoustical phonons (the only translational $q \approx 0$ modes). In other words; the absence of a far-infrared (external phonon) spectrum [15] should correlate with the absence of an externalphonon exciton sideband. In reality [14], this sideband extends over more than $100 \mathrm{~cm}^{-1}$, as do the rotational Raman bands [15] (there still are transla: tional $q \neq 0$ features). We therefore conclude that these data contradict Reynolds' approach. Also, results just obtained for benzene again do not fulfil his expectations. The authors of five recent papers on the benzene phonon sidebands $[16-20]$ all compare it to the phonon density-of-states [21] and not to the well known infrared modes [22]. This, again, does not mean that for some other systems his claim II may not hold true. We only submit that it is not justified, in general, and has not been borne out so far by the few systems which have been studied thoroughly:

\section{References}

[1] R. Kopelman, F.W Ochs and P.N. Prasad, J. Chem. Phys. 57 (1972) 5409.
(2) G.S Pawley, Phys. Stat. Sol. 20 (1967) 347 .

[3] P.A. Reynolds, J.K. Kjemis and J.W: White, J. Chem. Phys: 56 (1972) 2928 .

[4] P.A. Reynolds, Chem Phys. Letters 22 (1973) 177.

[5] P.N. Prasad and R. Kopelmen, J. Clem. Phys. 57 (1972) 863.

[6] M. Ito, AL Suzuki and T. Yokoyama, in: Excitons, magnons and phonons, ed. A.B. Zahlan (Cambridge Univ. Press, London, 1968) p. 1 .

[7] A. Hadni, B: Wyncke, G. Morlot and X. Gerbaux, J. Chem. Phys: 51 (1969) 3514.

[8. Aree and R.A. Kydd, Spectrochim. Acta 26A (1970) 1791.

19 F.W. Ochs, PhD. Thesis, Univ, of Michigan (1974).

[10 T. Azumi and Y. Nakano, J: Chem. Phys: 51 (1969) 2515

111 M. Ito and T, Shigeoka, J. Chem. Phys. 44 (1966) 1001.

[12] B.W. Gash, D.B. Hellman and S.D. Colson; Chem. Phys. 1.(1973) 1.91;

S.D. Colson and G.L. Wheeler, private communication.

[13 S.L Gaudioso, Ph.D. Thesis, Univ. of Michigan (1972).

[14] S.D. Woodruff, P.N. Prasad and R. Kopelman, J. Chem. Phys. 60 (1974) 2365.

[15] P.N. Prasad, S.D. Woodruff and R. Kopelman, Chem. Phys, 1 (1973) 173 .

[16] V.L Broude and V.K. Dolganov, Soviet Phys. Solid State 14 (1972) 225:

[17] V.K. Dolganov and E.F. Sheka, Soviet Phys. Solid State 15 (1973) 576 .

[18] Y.K. Dolganov, F.B. Slobodskai and E.F. Sheka, Soviet Phys Solid State 15 (1973) 595.

[19] F.B. Slobodskoi and E.F. Sheka, Soviet Phys. Solid State 15 (1973) 860 .

[20] S.D. Colson, T.L. Netzel and J.M. van Pruyssen, private communication.

[21] H. Bonadeo and G: Taddei, J. Chem. Phys. 58 (1973) 979.

[22] I. Harada and T. Shimanouchi, J, Chem. Hhys. 55 (1971) 3605 . 